\title{
Capsule Commentary on Blackstock et al., A Cross-Sectional Online Survey of HIV Pre-Exposure Prophylaxis Adoption among Primary Care Physicians
}

\author{
Natasha Parekh, MD \\ J Gen Intern Med 32(1):90 \\ DOI: $10.1007 / \mathrm{s} 11606-016-3915-8$ \\ (c) Society of General Internal Medicine 2016
}

University of Pittsburgh School of Medicine, Pittsburgh, PA, USA.

$\mathrm{T}$ he study by Blackstock et al. ${ }^{1}$ is a cross-sectional survey of primary care physicians (PCPs) regarding their selfreported awareness, adoption, and attitudes associated with adoption of HIV pre-exposure prophylaxis (PrEP). Although $93 \%$ of respondents reported awareness of PrEP, only $34.9 \%$ reported PrEP adoption, defined as ever having prescribed or referred a patient for PrEP. Compared with PrEP non-adopters, adopters were more likely to care for HIV-positive patients, report better knowledge about PrEP, and perceive PrEP as extremely safe. Compared with adopters, non-adopters were more likely to perceive PrEP use as leading to risk compensation.

While this article provides important insight into prescribing patterns and attitudes of PCPs, it has some confounders. First, the sample may not be fully generalizable given the low response rate $(8.6 \%)$ and potential sampling bias, since the participants comprised a convenience sample of primarily academic PCPs who were members of the same academic professional society. However, the authors note that the response rate was similar to those in previous studies on the topic. ${ }^{2,3}$ Second, the survey focused on self-reported knowledge about PrEP and its side effects. While self-reported knowledge is helpful for assessing confidence and comfort in prescribing PrEP, it may not translate into actual knowledge about PrEP and its side effects.

This article has important clinical implications. First, the fact that the rate of PrEP adoption (35\%) was among the highest in the literature is promising, suggesting that with time, adoption will continue to increase as PCPs become more

aware of and comfortable with PrEP screening and initiation. Given that the majority of the 1.2 million individuals in the USA who are eligible for PrEP have not initiated it, ${ }^{4}$ and that most PCPs are aware of PrEP, ${ }^{1}$ it will be important to identify precisely where the bottleneck is occurring in the clinical process of prescribing PrEP (i.e., screening for eligibility, counseling about benefits and risks, prescribing/referring, monitoring). Future studies should investigate where barriers to prescribing might arise and should assess interventions targeting these steps. Furthermore, as institutions begin developing PrEP protocols to streamline prescribing processes, reassessment of PrEP adaption will be imperative.

Corresponding Author: Natasha Parekh, MD; University of Pittsburgh School of Medicine, Pittsburgh, PA, USA (e-mail: nkp10@pitt. edu).

Compliance with Ethical Standards:

Conflict of Interest: The author has no conflict of interest with this article.

\section{REFERENCES}

1. Blackstock OJ, Moore BA, Berkenblit GV, et al. A cross-sectional online survey of HIV pre-exposure prophylaxis adoption among primary care physicians. J Gen Intern Med. 2016. doi:10.1007/s11606-016-3903-z.

2. Krakower DS, Oldenburg CE, Mitty JA, et al. Knowledge, beliefs and practices regarding antiretroviral medications for HIV prevention: results from a survey of healthcare providers in New England. PLoS One. 2015;10, e0132398.

3. Adams LM, Balderson BH. HIV providers' likelihood to prescribe preexposure prophylaxis (PrEP) for HIV prevention differs by patient type: a short report. AIDS Care 2016:1-5.

4. Smith DK, Van Handel M, Wolitski RJ, et al. Vital signs: estimated percentages and numbers of adults with indications for preexposure prophylaxis to prevent HIV acquisition-United States, 2015. MMWR Morb Mortal Wkly Rep. 2015;64:1291-5.

Published online November 10, 2016 